\title{
El De angelo perdito de Gilberto Crispino frente al De casu diaboli de Anselmo de Canterbury: convergencias y divergencias
}

\author{
NATALIA JAKUBECKI \\ Universidad de Buenos Aires \\ Consejo Nacional de Investigaciones Científicas y Técnicas
}

Resumen: El De angelo perdito, escrito por Gilberto Crispino circa 1094 y sin duda nacido del círculo de pensamiento anselmiano, es un texto a tal punto cercano al De casu Diaboli que la historiografía ha tendido a verlo como una copia de menor calidad o una especie de síntesis de este. Sin embargo, dicha convicción está fundada en una lectura un tanto superficial. De allí que este artículo tiene por objeto analizar las premisas y argumentos centrales de ambas obras con el fin de establecer con mayor precisión hasta dónde llega la innegable influencia de Anselmo y cuáles son, por el contrario, los aportes originales de Crispino.

Palabras clave: voluntad, razón, pecado, demonio, caída. 


\title{
The De Angelo Perdito by Gilbert Crispin Compared with the De Casu Diaboli by Anselm of Canterbury: Convergences and Divergences
}

\begin{abstract}
The De Angelo Perdito, which was written by Gilbert Crispin circa 1094 and doubtless belongs to the Anselmian thought circle, is close to De Casu Diaboli to such an extent that historiography has tended to see it as a lower quality copy or a kind of summary of it. Nonetheless, that conviction is founded on a superficial reading. With that in mind, this paper aims to analyse the central premises and arguments of both works in order to determine on one side Anselm's undeniable influences and, on the other, Crispin's original contributions.
\end{abstract}

Key-words: will, reason, sin, devil, fall.

—encuentra íntimamente ligado al de Anselmo de Canterbury, al punto que es casi imposible no leer los escritos de quien fuera el cuarto abad de Westminster a la luz de los de su amigo y antiguo maestro en Bec. Sin embargo, en el afán de poner de relieve la innegable influencia que Anselmo ejerció sobre uno de sus discípulos más destacados, la crítica del siglo pasado no pocas veces ha menoscabado -cuando no directamente malinterpretadolos escritos de Gilberto. ${ }^{1} \mathrm{Y}$ aunque en los últimos años se ha emprendido el camino de enmendar antiguas falencias hermenéuticas, por lo general los trabajos se han limitado al estudio de sus dos obras más conocidas, la Disputatio iudei et christiani (DCJ) y la Disputatio christiani cum gentili (DCG) (véase v.g. Goebel 2012; Whidden 2015). Con la intención de contribuir a este proceso de revisión y, al mismo tiempo, ampliar el espectro de obras crispinianas estudiadas, este artículo tomará como eje de análisis las principales tesis del De angelo perdito (DAP) -breve tratado en forma de dialogo entre las figuras de Responsio e Interrogatio escrito circa 1095 y que, como su título permite sospechar, trata sobre la caída del Diablo- y las comparará con las del De casu Diaboli (DCD) de Anselmo.

Si bien hay evidencia para suponer que Gilberto podría no haber leído el $D C D$ en su versión final (véase Evans 1986: xxxiv), el problema

\footnotetext{
${ }^{1}$ Uno de los ejemplos más representativos de las injusticias hermenéuticas de las que ha sido objeto Crispino es un artículo de Southern en el cual el autor se las ingenia para quitarle todo mérito posible en favor de Anselmo. Este caso está analizado en Jakubecki 2018: 9-11.
} 
no consiste en determinar qué pudo o no haber leído, sino en precisar los alcances y límites de una fuente de inspiración que de ningún modo puede ser puesta en duda como tal. De hecho, Gillian Evans, eximia estudiosa del pensamiento anselmiano y una de las responsables de la edición crítica del $D A P$, ha afirmado que esta obra es una simplificación deliberada del $D C D$ para evitar las dificultades filosóficas de una obra a la que, de todas maneras, sigue "punto por punto y tópico por tópico" (1986: xxxiv). Posiblemente esta sea la razón por la que -al menos hasta donde hemos tenido noticias- no existe ningún trabajo consagrado íntegra o parcialmente al $D A P$, y son contados aquellos que le dedican algunas líneas, por lo general relacionadas con el texto anselmiano. No obstante, fue la misma Evans quien anteriormente había sostenido, también a propósito del $D A P$, que en él Gilberto "no repitió simplemente a Anselmo, sino que [...] produjo un tratado que se sostiene por sus propios méritos" (1980: 201).

Ahora bien, el $D A P$ puede ser dividido en dos grandes partes. La primera, que comprende las secciones 1-42, es un único y gran bloque que problematiza diferentes aspectos de la caída del Diablo y cuyas fuentes principales -sino exclusivas- son las obras que Anselmo escribió en Bec en especial, naturalmente, el DCD. La segunda parte, que va desde la sección 43 hasta la 82 , es decir, hasta el final, es más bien un conjunto de reflexiones sobre diferentes tópicos que, aunque están vinculados con la caída y/o se tratan en el DCD, son relativamente independientes unos de otros. Esta pérdida de organicidad se refleja también en las fuentes, que en esta segunda parte se amplían al menos a Agustín y Boecio. En efecto, las secciones 43-48 tratan de cómo ha de entenderse "posse" en relación con la omnipotencia divina, tema que Anselmo trabajó en varias de sus obras. Las secciones 49-56 discurren acerca del problema de la providencia divina en relación con el libre albedrío, el cual Anselmo apenas esboza en DCD 21 y recién trata detenidamente en el De concordia. En estas secciones, Gilberto sigue a Boecio (véase Jakubecki 2018). Las secciones 57-63 versan sobre la restitución del número de la ciudad celestial luego de la caída de los ángeles, tópico que, aunque tematizado en Cur Deus Homo I.16-18 (obra posterior al DAP), es propio de la tradición agustiniana y recurrente en los opúsculos teológicos del siglo XI (véase Novotný 2014: 14-40). Por último, desde la sección 64 hasta el final, Crispino explica cómo debe entenderse la unidad de Dios y la unidad de la creatura. Una vez más -y aunque allí el apparatus fontium de la edición crítica enmudezca por completo- la principal fuente es Boecio, al que incluso cita de manera textual. Teniendo en cuenta esta estructura temática, nuestro análisis se circunscribirá a la primera parte pues es precisamente aquella en la que la originalidad de nuestro autor respecto de la doctrina anselmiana resulta menos evidente. 
Para cumplir de manera cabal nuestro propósito hemos dividido el presente artículo en tres grandes ejes, cada uno de ellos dado por el desarrollo problemático de un pasaje bíblico común al $D A P$ y el $D C D$. El primero se enfoca en cómo cada autor piensa las nociones de verdad, justicia y felicidad a partir del pasaje de Juan 8:44 según el cual el Diablo "no permaneció en la verdad" (in veritate non stetit). El segundo eje, titulado con la pregunta de Pablo en I Corintios 4:7: “¿Qué tienes que no hayas recibido?" (Quid autem habes quod non accepisti?), está dividido en dos momentos. El primero de ellos versa sobre el don de la perseverancia y la responsabilidad de Dios en la caída del ángel; el segundo, sobre el papel de la razón, la voluntad y el libre albedrío en relación con la comisión del pecado. Finalmente, el tercer y último eje se centra en la exégesis que cada autor hizo del pasaje de Isaías 14:14: "seré como el Altísimo" (similis ero Altissimo).

Resta advertir que, a pesar de la índole comparativa de este artículo, el análisis se centrará en el $D A P$ y no el $D C D$, pues no solo es el texto menos conocido y estudiado sino que es sobre él en donde recae el "onus probandi". En contrapartida, necesariamente deberemos resumir algunos aspectos de la doctrina anselmiana que no en vano ha sido y continúa siendo objeto de sinnúmero de trabajos académicos. Desde luego, ello no impedirá que justifiquemos nuestra posición y consignemos las piezas bibliográficas pertinentes toda vez que lo creamos oportuno.

\section{In veritate non stetit}

E $\mathrm{n}$ sus respectivos tratados sobre la caída del Diablo, tanto Antención: explicar un dato de fe que, como tal, ya han asumido. Mientras que, a partir de la exégesis bíblica tradicionalmente aceptada dan por sentado que fue un ángel la primera creatura en pecar, lo que se proponen es explicar, dicho brevemente, por qué y cómo fue posible ese pecado. Crispino no duda en tomar como punto de partida para su planteo el mismo pasaje que en el segundo capítulo del DCD había dado lugar al desarrollo problemático de la caída, aquel del evangelio de Juan en el que el apóstol afirma que el Diablo "no permaneció en la verdad" (Jn. 8: 44). Esta elección ya en el mismo comienzo del $D A P$ pone de manifiesto que el tratado posee una fuerte inspiración anselmiana. Con todo, es justamente en qué entiende cada autor por "stare in veritate" donde encontramos la primera divergencia entre ellos.

En su primera intervención, Interrogatio dice: 
estar en la verdad es saber que lo que es, es, y que lo que no es, no es. Porque estimar que es lo que no es, o que no es lo que es, no es estar en la verdad sino estar en la falsedad. El ángel perdido, entonces, estuvo en la verdad tanto cuanto estuvo en el conocimiento de la felicidad [...] Pero el que no quiso permanecer en la felicidad, ignoraba, en efecto, en qué consiste la felicidad. ¿Pues quién en su sano juicio no quiere estar en la suficiencia de su voluntad? $(D A P 1-3)^{2}$

A nadie que conozca el De veritate se le oculta el hecho de que la definición de verdad que formula este personaje -y Crispino, en realidad, pues en ningún momento del texto es contradicha por Responsio sino, antes bien, retomada (véase $D A P 21$ ) - es apenas diferente de la que en el tercer capítulo de aquel texto había ofrecido Anselmo a propósito de la verdad del pensamiento (veritas cogitationis): cuando "juzgamos (putamus) que es lo que es, y que no es lo que no es”. Pero aquí Crispino no solo está definiendo el concepto de verdad sino que, además, está explicando qué ha de entenderse por "estar en" (esse in) la verdad o la falsedad.Y ese "estar" es equivalente a un determinado estado epistémico: el de saber (cognoscere) en el primer caso; el de estimar o creer (estimare) en el segundo. Desde luego, esta diferencia nos deja entrever que Crispino no es ajeno al principio parmenídeo según el cual no se puede conocer lo que no es. Sea de ello lo que fuere, lo cierto es que para este autor la verdad está ligada especialmente a la actividad intelectual.

Luego de haber explicado esto, Interrogatio realiza un desplazamiento desde "stare in veritate" a "stare in beatitudine" -término que Responsio retomará en más de una oportunidad (véase DAP 14, 16 y 18)-. Al mismo tiempo, por medio de una pregunta retórica, explica que estar en la felicidad es estar en la "suficiencia de la voluntad", esto es, de una voluntad que no quiere nada más que lo que tiene porque tiene todo lo que quiere. Por consiguiente, podemos decir que, para Crispino, la felicidad es a la voluntad lo que la verdad es al entendimiento, y a su vez ambas, verdad y felicidad, son equivalentes entre sí.

\footnotetext{
${ }^{2}$ Las obras de Crispino se citan por número de sección de la edición crítica de Abulafia y Evans 1986; y las obras de Anselmo se citan por número de capítulo según la edición crítica de F. S. Schmitt 1946. Todas las traducciones de texto latino y los resaltados son propios, salvo expresa indicación en contrario. En el caso de la Disputatio Christiani cum Gentili se agrega, además, número de página de la traducción al castellano de Jakubecki en Crispino 2017.
} 


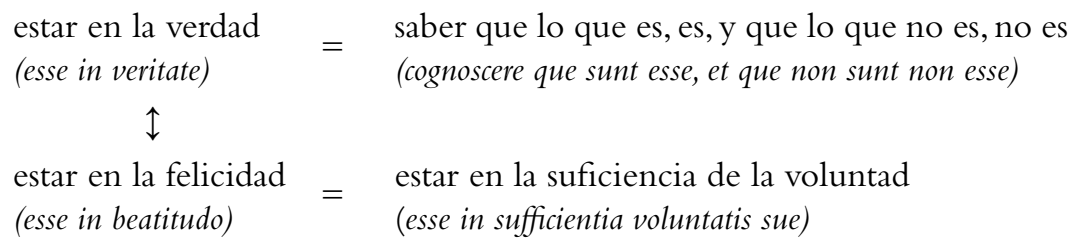

Ahora bien, ese objeto que satisface por completo la voluntad es la posesión, el conocimiento de una verdad muy concreta que se hace explícita cuando, más avanzado el texto, Responsio afirma que "Dios le dio al Diablo el [...] poder permanecer en esta verdad (in hac veritate): que Dios es mayor que él, y él menor que Dios; pero no quiso perseverar en esta verdad en la que estaba" (DAP 21). Así pues, al menos en el caso de los ángeles en general, y del Diablo en particular, estar en la verdad equivale a saber que ellos son inferiores respecto de Dios, y estar en la felicidad -o, lo que es lo mismo, ser feliz- es querer que así sea.

En Anselmo, en cambio, desentrañar qué significa el "stare in veritate" es una cuestión un poco más compleja y para resolverla debemos remitirnos al De veritate, obra cuya lectura, según el propio autor, está supuesta en el $D C D$ (véase $D V$ praefatio). ${ }^{3}$ Como buen platónico-agustiniano, Anselmo entiende que todo lo que es, es o se dice verdadero en relación con la Verdad única y suprema que es Dios o el esse. Sin embargo, aun cuando la verdad sea una sola, desde el punto de vista de la multiplicidad creada se puede hablar, aunque impropiamente, de diferentes verdades o tipos de verdad según ella resida en la enunciación, el pensamiento, la voluntad, la acción o la esencia de la cosa (véase $D V 10$ y 13$).^{4}$

Anselmo también establece allí una suerte de correspondencia entre verdad, rectitud y justicia, de modo que ellas "se definen mutuamente" ( $D V$ 12). La verdad es equivalente a la rectitud en tanto se define como "rectitudo mente sola perceptibilis" (DV 11), y la rectitud, por su parte, no es sino debitum, lo que cada cosa debe ser o hacer según su propia naturaleza. ${ }^{5} \mathrm{Y}$ la justicia, finalmente, es una "rectitudo voluntatis propter se servata" (DV 12), es decir, una especie de rectitud que concierne concretamente al horizonte volitivo. Así,

\footnotetext{
${ }^{3}$ Véase también Briancesco 1978: 120-124.

${ }^{4}$ Aquí hemos simplificado deliberadamente la relación entre la verdad suprema y las verdades relativas en función de nuestro interés principal: esclarecer qué entiende Anselmo por "stare in veritate”. Para una explicación pormenorizada puede consultarse Dreyer 1997 y Corti 2011. ${ }^{5}$ Este debitum posee un sentido ontológico (lo que cada cosa debe ser) y un sentido moral (o hacer) que no corresponde analizar aquí. Sobre ellos véase Corti 2013: 80-85.
} 
verdad y justicia son equivalentes en lo que respecta al ámbito moral, propio de las creaturas racionales, porque ellas son, precisamente, las únicas que pueden percibir y querer esa rectitud en la que tanto la verdad como la justicia consisten.

Notemos que, al igual que para Crispino, la verdad para Anselmo también está ligada al horizonte intelectual, dado que no es cualquier tipo de rectitud, sino únicamente aquella perceptible mente sola. Sin embargo, mientras Crispino basa su comprensión del pasaje de Juan en la definición anselmiana de la verdad del pensamiento, Anselmo dice explícitamente en el De veritate 4 y 5 que esa verdad a la que se refiere el evangelista se trata de la verdad de la voluntad (veritas voluntatis) y, por tanto, de una cuestión eminentemente moral, no epistémica.

Según Anselmo, pues, "estar en la verdad" es estar en posesión de una voluntad recta, es decir, una voluntad que quiere lo que debe (véase $D V 4)$.Y eso que debe querer no es sino la misma rectitud o justicia, ese bien autónomo y superior respecto de cualquier otro bien que, por tanto, se debe querer por sí mismo (propter se) y no porque hacerlo sea lo debido (qua debitum) ni, mucho menos, con vistas a algún otro bien (véase Zoppi 2009: 28). ${ }^{6}$ De ahí que en el DCD, "stare in veritate", "stare in rectitudine" y "stare in iustitia" resulten ser expresiones intercambiables (véase 9 et passim). Pero no sucede lo mismo con el stare in beatitudine, expresión que, de hecho, Anselmo jamás utiliza.

Aunque en Dios, dada su simplicidad, la justicia no difiere de la felicidad (véase $D C D$ 12), en lo que toca a la creatura estos son dos bienes distinguibles: la felicidad, que proviene del bienestar o conveniencia (ex commodis), es un bien que nada dice de la condición moral en tanto es naturalmente anhelada por toda creatura sensible (véase DCD 4 y 12); la justicia, en cambio,"es el bien mismo por el que los buenos son buenos" (DCD 8). Pero no solo son bienes diferentes sino que entre ellos existe una jerarquía dada por su valor moral intrínseco: la felicidad está en cierto modo supeditada a la justicia pues mientras la justicia es ese bien que los seres racionales deben querer por sí mismo, la felicidad es la retribución que reciben por ser justos; aunque esta retribución no es ya mera beatitudo ex commodis sino beatitudo cum iustitia (véase DCD 12; Zoppi 2009: 30 y Goebel 2010).

Por otra parte, el hecho de que felicidad y justicia no coincidan en la creatura es lo que da lugar al conflicto y hace del ángel un agente moral.

\footnotetext{
${ }^{6}$ De hecho, en este "propter se" se disuelve la oposición entre autonomía y heteronomía, pues justamente es la superación del debitum qua debitum. Corti 2013: 84; Briancesco 1978: 128-129.
} 
Tal como explica Anselmo, los ángeles fueron creados tanto con la voluntad o tendencia a la justicia como con la voluntad o tendencia a la felicidad. ${ }^{7}$ Aunque igualmente naturales, estas voluntates eran capaces de entrar en tensión y, de hecho, en la economía teórica anselmiana es necesario que puedan hacerlo pues de otro modo no serían posibles ni el pecado ni el mérito. ${ }^{8}$ Cuando el Diablo antepuso el deseo de felicidad al de justicia, pecó porque invirtió la relación entre estos dos bienes.

Para Anselmo, entonces, stare in veritate es sinónimo de stare in iustitia y a su vez, stare in iustitia implica, aunque no lo diga en estos términos, stare in beatitudine. Pero, a diferencia de Crispino, en este último par no hay una equivalencia sino una relación de subordinación: si, o mejor dicho, porque se está in iustitia (porque se es justo), se está en beatitudine (se es feliz), pero no viceversa. Ello se hace evidente en el caso de Diablo pues fue queriendo aumentar su felicidad que abandonó ipso facto la justicia y, por tanto, dejó no solo de ser feliz sino también de permanecer en la verdad en la que se hallaba.

Esta subordinación, sin embargo, tiene lugar en la condición prelapsa. Como señala Díez, la tensión entre la voluntad de justicia y de felicidad se resuelve después de la caída, cuando los ángeles son dotados de un nuevo conocimiento que antes ni tenían ni podrían tener: que estas dos "afecciones de la voluntad son 'una y la misma' y que 'amar ser justo' es la beatitud a la que todas las creaturas racionales están llamadas”, (2014: 104). Así, aquella suficiencia de la voluntad que, según Crispino, todos los ángeles poseían por igual en su estado natural, según Anselmo solo será posible para los ángeles confirmados que, al haber conquistado la beatitudo cum iustitia, ya no desean sino únicamente lo que tienen (véase DCD 6).

\footnotetext{
7 Aun cuando en los capítulos 12-14 del DCD Anselmo habla de "voluntas beatitudinis" y "voluntas iustitiae", debe entenderse que aquí está hablando de disposiciones o tendencias. De hecho, el mismo autor aclarará, años después que "voluntas" puede referirse a la facultad que quiere, a la tendencia de esa facultad hacia un objeto, o a la acción misma de querer. Véase Visser y Williams 2006: 188.

${ }^{8}$ Coincidimos con Vanni Rovighi cuando sostiene que mientras la tendencia a la felicidad es natural y legítima en todos los animales no racionales, para las creaturas racionales resulta peligrosa porque, de alguna manera, es una tentación contra su virtud propia que es tender a la justicia (1969: 86-87). Goebel, por su parte, sostiene que la tradicional lectura que entiende la tendencia a la felicidad y la tendencia a la justicia como antagónicas es errónea, si bien es cierto que el propio vocabulario de Anselmo por momentos se presta a confusión (2010: 92 ss).
} 


\section{Quid autem habes quod non accepisti?}

\subsection{Perseverancia del ángel y responsabilidad de Dios}

En el $D A P$, la afirmación de que el ángel no permaneció en la verdad dispara en Interrogatio la pregunta de por qué (quare) no lo hizo. Entonces propone dos respuestas posibles: o bien porque no pudo, o bien porque no quiso (véase $D A P$ 2), y luego pasa a explorar qué se sigue de cada una de ellas. Si no quiso, podría pensarse que el Diablo ignoraba en qué consiste la felicidad pues nadie "en su sano juicio" (non amens) quiere no ser feliz (DAP 3). Sin embargo, de ello se seguiría que "fue creado necio e ignorante de tan grande posesión" y, en consecuencia, que "no fue creado feliz sino desdichado y miserable" (DAP 4). Pero esto no puede ser ya que, de ser así, jamás habría estado en la verdad en la que dejó de estar y, por tanto, en modo alguno el Señor lo habría culpado de no permanecer allí donde nunca estuvo. Interrogatio, entonces, concluye que si no quiso fue, sencillamente, porque "no tuvo libre albedrío de la voluntad" (DAP 7). En cambio, si quiso pero no pudo, debió ser porque, aun gozando del libre albedrío, habría sufrido "alguna violencia contra [este] que oprimió la capacidad de la razón" (DAP 7). Así, sea cual fuere la respuesta, en definitiva, se llega a la misma conclusión: el Diablo, dice Interrogatio, "no pudo querer lo que era necesario querer, esto es, permanecer en la verdad" (DAP 7). De lo cual se sigue que el Diablo era impotente, y por tanto inimputable, y por tanto inocente, lo cual contradeciría el dato de la fe. Pero no solo eso.

En medio de su planteo, Interrogatio evoca el versículo paulino: “¿Qué tienes que no hayas recibido?" (I Cor. 4: 7), con el que Crispino pretende poner de manifiesto que ninguna creatura tiene nada por sí, sino que todo lo que tiene lo recibe de Dios, y que todo lo que se recibe es un bien "pues todo lo bueno existe por Dios" (DAP 9).Y si bien este pasaje es el mismo que Anselmo problematiza en el primer capítulo del $D C D$, en el $D A P$ se omite el análisis acerca de los usos equívocos de "facere", soslayando, así, el problema de la deuda ontológica de la creatura respecto del creador (factor-factum) y el de su proveniencia y tendencia a la nada originaria (aliquid-nihil), ambos fundamentales para comprender el desarrollo problemático del DCD. Lo que a Crispino le interesa no es el aspecto ontológico del debitum sino el moral. Es por eso que pasa a señalar el núcleo de la cuestión sin ningún tipo de preámbulo: para que el Diablo pudiera haber permanecido en la verdad debió haber tenido perseverancia en el bien; pero dado que no permaneció, no la tuvo. Interrogatio, entonces, comete el mismo error que había cometido el Discipulus de Anselmo. Establece un falso paralelismo entre tener/recibir y no tener/no recibir, a partir del cual deduce que si todo lo que las crea- 
turas tienen lo han recibido de Dios, entonces todo lo que las creaturas no tienen, no lo tienen porque Dios no se lo dio (véase $D A P$ 6; DCD 2-3). En el tratado de Anselmo es recién en el tercer capítulo cuando aparece la disyuntiva de si el Diablo no pudo o no quiso, no ya permanecer en la verdad, como preguntaba Interrogatio, sino si no pudo o no quiso recibir la perseverancia. De todas maneras, la pregunta apunta al mismo problema. Ahora ya no solo sucede que el Diablo parece ser inimputable, sino que, además, Dios sería el responsable de la caída al no darle al ángel lo que necesitaba para no caer, esto es, la perseverancia para permanecer en la verdad.Y a ello se le sumaría el hecho de que habría sido injusto al condenarlo dado que le exigió lo que no podía.

Anselmo, entonces, explica que no tener no necesariamente implica que no se haya dado, sino que bien puede ser consecuencia de no haber querido recibir, tal como sucedió en el caso del Diablo. Gilberto, en cambio, aunque de alguna manera da por sentada esta explicación cuando dice al pasar: "y no la recibió no porque Dios no se la diera, [...] sino porque no quiso conservar lo que recibió" (DAP 20), opta en primer lugar por resolver la cuestión a través de dos distinciones centrales realizadas por Responsio.

La primera de ella es que, al igual que sucede con el uso de la razón, ${ }^{9}$ perseverar no es lo mismo que poder perseverar, sino que toda acción efectiva -el "acto", si se nos permite expresarnos en términos aristotélicossupone, antes, el poder -la "potencia"- de realizar esa acción, incluso si "no usa la razón todo el que puede usarla, pues a veces abusa; y no persevera todo el que puede perseverar" (DAP 11). La segunda distinción versa sobre los alcances y modos de ese poder:

Y una cosa es poder o no poder usar la razón, y otra cosa es poder usar o no usar la razón. Pues aquel que no puede usar la razón, es irracional o no racional; en verdad se puede ser racional incluso si alguna vez se abusa de la razón. Así pues, una cosa es poder o no poder perseverar, y otra es poder perseverar o no perseverar. (DAP 12)

Aunque de un modo más retórico, lo que está intentando decir Crispino es similar a lo que Anselmo había dicho a través de una corrección que el Maestro le hace al Discipulus en el tercer capítulo del DCD: "no dije que Dios le dio el recibir la perseverancia, sino solo el querer y poder de recibir la perseverancia". Pero si hemos dicho "similar", es porque Crispino,

\footnotetext{
${ }^{9}$ Nos detendremos en este paralelo más adelante.
} 
una vez más, no está siguiendo al pie de la letra las enseñanzas de su mentor. Según Crispino, lo que Dios le dio al Diablo y de hecho el Diablo recibió y tuvo no fue la perseverancia en sí misma, sino el poder de perseverar en el bien (véase DAP 21)..$^{10}$ Para Anselmo, Dios tampoco le dio la perseverancia en sí misma sino el velle -entiéndase, la voluntad-y el posse -entiéndase, el libre albedrío- por medio de los cuales puede no directamente perseverar, como propone Crispino, sino recibir y querer recibir la perseverancia; esa misma que efectivamente recibieron los ángeles buenos (véase $D C D$ 4).

A pesar de esta ligera diferencia, el problema de la imputabilidad del Diablo y la responsabilidad de Dios por su caída respecto de la perseverancia se resuelve de la misma manera tanto en el $D A P$ como en el $D C D$ : Dios no es en modo alguno responsable pues dotó al ángel de lo que le era necesario para perseverar, y por tanto tampoco fue injusto castigándolo, pues le exigió algo que sí podía. Con todo, en el DCD, las sucesivas preguntas del Discipulus vuelven una y otra vez a poner en discusión la responsabilidad de Dios, por lo que esta cuestión no se terminará de zanjar sino hasta el final de la obra: dado que todo lo que es proviene de Dios, y que el querer del Diablo era algo, Anselmo debe concluir que, aunque Dios no aprueba el uso indebido de este querer, lo permite.Y en este sentido, según el análisis de los modos en que se entiende "hacer" que consta en el primer capítulo del $D C D$, se puede decir, aunque de modo impropio, que Dios hace el mal porque no hace que no sea. En cambio, desde el momento en que Crispino omite el análisis sobre las modalidades del facere, no hay lugar en su obra para que su argumentación derive en esta conclusión. ${ }^{11}$

\subsection{El pecado y el libre albedrio: entre la razón y la voluntad}

En el apartado anterior hemos visto que ambos autores coinciden en que Dios no le dio al Diablo la perseverancia sino, en el caso de Anselmo, el poder y querer recibirla, en el caso de Crispino, el poder de perseverar. Detrás de esta "ligera diferencia" -como la hemos llamado- se encuentra, en realidad, el mayor distanciamiento de Crispino respecto de Anselmo.

\footnotetext{
${ }^{10}$ Esto soluciona también la aparentemente contradictoria explicación de Responsio cuando afirma que el ángel "no tuvo (non habuit) perseverancia en el bien [...] no porque Dios no se la diera (pues Dios dio y él recibió) sino porque no quiso perseverar en eso que recibió y que tuvo (et habuit)", DAP 10.

${ }^{11}$ Sobre las modalidades de facere, tema que no resulta pertinente desarrollar aquí, puede consultarse Castañeda 2005: 31-38.
} 
Atendamos, una vez más, a las dos distinciones que realiza Crispino, pues junto a la perseverancia aparecen otros varios elementos de interés. El primero de ellos es el "poder y poder no". Esa doble modalidad de "poder" se debe a la potencia del libre albedrío, gracias a la cual, dirá Crispino en su DCG "se puede realizar cualquiera de las dos (utrumlibet) acciones antes que solo una de ellas" (16:18). Las acciones a las que se refiere en ese texto son, por una parte, pecar o no pecar, por otra, usar la razón o no usarla. En el $D A P$ "pecar o no pecar" es sustituido por "perseverar o no perseverar", pero ello es solo porque en lugar de referirse al pecado en general, Crispino alude al quomodo, a la naturaleza específica del pecado del ángel.

Este primer elemento se trata, entonces, de ese mismo posse que, según Anselmo, Dios le dio al ángel para que fuera capaz de recibir la perseverancia. Dado que en el primer capítulo de su De libertate arbitrii Anselmo se encarga de dejar en claro que " de libertad de arbitrio”, quizá pueda parecer que ambos autores tienen una concepción diferente del libre albedrío. Pero ello no es así. Según el arzobispo, una cosa es la libertad que posee el albedrío (libertas arbitrii), que es el "poder de conservar la rectitud de la voluntad por la rectitud misma" (DLA 3) - por lo que necesariamente y por definición excluye el poder de pecar (potestas peccandi) - y otra cosa es el libre albedrío (liberum arbitrium) que, no en tanto libre sino en tanto albedrío, permite, en palabras de Crispino, realizar cualquiera de las dos acciones. ${ }^{12}$ De allí que Anselmo explique la caída del ángel diciendo:

Pecó, pues, por su propio arbitrio, que era libre; pero no por aquello por lo cual era libre, esto es, por la potestad por la cual podía no pecar y no servir al pecado, sino que pecó por la potestad que tenía de pecar, por la cual ni fue ayudado en dirección de la libertad de no pecar ni fue obligado hacia la servidumbre del pecado. (DLA 2)

Y aunque Crispino no utiliza el término "libertas" en el $D A P$, aquella concepción de raigambre agustiniana de la que parte Anselmo según la cual la libertad consiste en poder no pecar, se hallará también en DCG 16 cuando explica que Adán "pudo no pecar pero quiso hacerlo". ${ }^{13}$ No es, por tanto,

\footnotetext{
${ }^{12}$ Sigo aquí la lectura clásica de Briancesco 1982: 59-66. Existen, con todo, muy variadas interpretaciones. Entre las más recientes se destaca la de Goebel, quien solo después de distinguir dos tipos de posse, puede sostener que "Le puvoir de pécher [est] impliqué par le pouvoir de ne pas pécher...” (2017: 94), resaltado del autor.

${ }^{13}$ En Crispino, la voz libertas aparece solo en dos obras (DCJ 101 y Semo in ramis palmarum
} 
en cuanto al posse que Gilberto se aleja de su mentor, sino en cuanto al velle.

Según explica Anselmo, el Diablo pecó porque no perseveró en su voluntad inicial de justicia. Ahora bien, perseverar es completar (perficere) una acción. Y como nada se puede completar sino queriendo hacerlo, y la acción propia de la voluntad es querer, "perseverar en la voluntad" es simplemente seguir queriendo hasta el final (o, lo que es lo mismo, hasta que Dios disponga) eso mismo que ya se quiere (véase $D C D$ 3).Y lo mismo parece entender Crispino por "perseverar" cuando, en la primera distinción, explica que "no persevera todo el que puede perseverar, pues muchas veces se aparta de lo que comenzó" (ab incepto, DAP 11), lo cual no es sino otra manera de decir que se deja de querer lo que se había comenzado a querer. No obstante, jamás habla de "perseverar en la voluntad", sino de "perseverar en el bien" (DAP 6, 10, 20 y 21) o, lo que para él es lo mismo dadas las equivalencias de la tradición platónico-agustiniana a la que pertenece, de "perseverar en la verdad" (DAP 21).

Aunque no está diciendo algo diferente de Anselmo -pues perseverar en la voluntad de justicia equivale a perseverar en el bien toda vez que la justicia no solo es un bien sino el bien supremo- llama la atención que omita cualquier mención ya sea de la potencia volitiva, es decir, la voluntad, ya sea de la acción propia de esa voluntad, el querer, y apunte directamente al objeto de ese querer. Lo que sucede es que la atención de Crispino no está puesta en la voluntad sino en el segundo elemento en el que toca reparar: la razón. En efecto, para él no se trata de si el Diablo pudo querer recibir la perseverancia, sino de que si pudo perseverar y pudo no perseverar fue porque, ante todo, era una creatura racional (véase DAP 13). ${ }^{14}$

Nótese que cuando Responsio distingue primero entre poder perseverar y perseverar, y luego entre poder perseverar y poder no perseverar, lo hace poniendo en paralelo la perseverancia con la razón. Ello es así porque para que el Diablo sea responsable no solo debió haber podido optar por cualquiera de las dos (utrumlibet) acciones, perseverar o no, sino también haber sido capaz de discernir lo justo de lo injusto, lo cual es posible me-

13-14 y 16) y en referencia a la condición prelapsa del hombre. En el Sermo, además, le adjunta el adjetivo "pristina", por lo que se puede inferir que se está refiriendo a la libertad agustiniano-anselmiana.

${ }^{14} \mathrm{Al}$ menos hasta donde hemos tenido noticias, Rakus ha sido el único que ha reparado en el énfasis que Crispino, a diferencia de Anselmo, pone en el uso de la razón respecto de la perseverancia. Sin embargo, con una mirada fiel a la tradición, relativiza su originalidad arguyendo que, de todas maneras, lo que el abad dijo se encuentra sugerido indirectamente en $D C D 5$ (1997: 121). 
diante la razón (véase DCG 6), facultad que, a su vez, puede usarse o no gracias a ese mismo libre albedrío por el que se puede perseverar o no.Y tal es la eficacia del libre albedrío sobre la razón que, en su tratado De anima $(D A)$, Crispino dice saber certissime que el alma "tiene libre albedrío porque puede usar y abusar de la razón" (4-5).

En el DAP nuestro autor se refiere a la ratio como un "munus" (3940), sustantivo que puede ser traducido tanto por "función", como por "obsequio" y por "obligación". La elección de este ambiguo término en modo alguno es casual. Para Crispino, la razón es un don cuyo uso es un deber, pues Dios creó a los seres racionales para que lo conozcan y, gracias a ello, le den lo que se le debe como creador: amor, temor y obediencia (véase $D A P$ 26 y 39 y DCG 7). De modo que si la creatura "no quiere usar el don de la razón [...], mejor dicho, si abusa, cometerá una injuria completa contra Dios" (DAP 40). Esta afirmación nos conduce al tercer y último elemento a considerar: el abuso.

El abuso está vinculado a la razón a lo largo de toda la obra de Crispino. Entre los pasajes que ya hemos citado, se alude al abuso de la razón no solo en aquel del $D A$ que da cuenta de la existencia del libre albedrío, sino también en las dos distinciones eje de la argumentación del $D A P$. Recordemos que en la primera Responsio había dicho que "no usa la razón todo el que puede usarla, pues a veces abusa", y en la segunda, que la creatura "puede ser racional incluso si alguna vez abusa de la razón". La única excepción en la que el abuso no está ligado a la razón es un pasaje del DAP en el cual Responsio afirma que el Diablo "pecó por el abuso de la potestad del libre albedrío" (DAP 17), pero ello es porque el libre albedrío es la potencia por cuyo ejercicio y existencia Interrogatio se había preguntado al comienzo de la obra, por lo que era necesario despejar toda duda al respecto. Pero incluso en aquel momento del argumento también aparece marcada la importancia de la razón en el acto libre. Habíamos visto que cuando Interrogatio explora las dos opciones por las que el Diablo pudo no haber permanecido en la verdad, concluye que o bien no tuvo albedrío o bien hubo alguna violencia contra este que oprimió no la voluntad -como cabría esperar si siguiera con exactitud la argumentación de Anselmo (véase DCD 16; Monologion 69)sino la potentia rationis.

Si hemos de buscar la definición de pecado que mejor dé cuenta del pensamiento de Crispino, ella es la que propone el personaje del Cristiano en la DCG a propósito del pecado de Adán quien "pecó por el abuso voluntario de la razón" (16:18). La similitud de este pasaje con DAP 17 es evidente. No obstante, esta fórmula es más completa porque hace hincapié en las dos facultades involucradas en el pecado -razón y voluntad-incluyendo tácita- 
mente y, a la vez, superando la mera potencia del libre albedrío. ${ }^{15}$ Más aún, que Crispino entiende al pecado como un abuso voluntario de la razón lo confirma, además, la recompensa de los ángeles buenos, que consiste en “ya no poder abusar de la razón” (DAP 20), es decir, en la eliminación no de la facultad racional, sino del modo de uso por el que ella puede pecar; recompensa que, por tanto, puede simplificarse diciendo que consiste en ya no poder pecar.

A primera vista puede parecer que se trata de la misma recompensa que Anselmo propone, la cual, a su vez, descansa en aquella libertad última por la que, según Agustín, se fortalece la voluntad de manera que solo puede orientarse hacia el bien. ${ }^{16}$

Agustín De correptione et gratia XII.33 non posse peccare

Anselmo DCD 6

Crispino $\quad D A P 20$ peccare nequeunt

iam peccare non posse

iam non posse abuti ratione

Por más que en todos los casos la recompensa consista en ya no poder pecar, Crispino se aparta de Agustín y Anselmo desde el momento en que reemplaza "pecar" por "abusar de la razón”, o sea, por la definición de pecado compuesta de la acción específica por la que se peca y la facultad con la que se lleva a cabo. En efecto, esta sustitución implica que es la razón y no la voluntad la facultad que se fortalece tras la confirmación de los justos así como también es ella la que, en contrapartida, se debilita con el castigo de los injustos (véase DAP 19).

${ }^{15}$ Aunque se refiere al pecado del hombre, es posible extrapolar esta definición también al pecado angélico dado que ambas creaturas fueron creadas iguales "según la gracia de la razón y la libertad concedidas" (DCJ 101). Algo similar ocurre en el caso de Anselmo quien, en la oración final de $D C D$ 7, después de hablar del Diablo, agrega que lo dicho "también puede cuestionarse sobre nuestra propia naturaleza".

${ }^{16}$ Anselmo habla de la recompensa de los ángeles buenos en similares términos en Cur Deus Homo I.18. Respecto del tópico de la libertad y el fortalecimiento o debilitamiento de la voluntad en Anselmo, Goebel sostiene que la intención del arzobispo no fue otra que la de "reconstituer la théorie augustinienne de la liberté et de la grâce dans une forme plus cohérente" (2017: 85). Para la tipificación del fortalecimiento de la voluntad en Anselmo véase ibíd.: 80-85. 


\section{Similis ero Altissimo}

Tolviendo la atención nuevamente al concepto de "abuso", ha de notarse que Anselmo en ningún momento emplea este término, pero sí, y en más de una oportunidad, el de "rapiña" (véase $D C D$ 8, 26 y 28). Mientras que la rapiña o el robo consiste en una apropiación indebida, el abuso puede tener una acepción cuantitativa, como uso desmedido, o bien cualitativa, como uso impropio, uno para un fin diferente de aquel que conviene a la naturaleza de aquello de lo que se abusa. Ahora bien, según esta última acepción -que es la que maneja Crispino y que es del todo afin al pensamiento anselmiano $-{ }^{17}$ es posible decir, como lo ha hecho Olivares, que la rapiña del Diablo se debió a un abuso (véase 1952: 679). En efecto, desde el instante en que el Diablo usó su voluntad no para lo que fue hecha (ad quod facta est) -es decir, querer la justicia- sino para querer sponte una felicidad que aún no debía tener, se apropió de su voluntad injustamente, incluso cuando esta apropiación haya sido permitida por Dios en tanto no hizo que no sea (véase $D C D$ 28).

De todas maneras, aunque es posible explicar el pecado del Diablo según Anselmo en términos de abuso, esto no es exactamente lo que Crispino entiende por pecado, pues él no habla de abuso de la voluntad sino de abuso voluntario de la razón. La voluntad, elemento central del análisis anselmiano, forma parte de la definición de pecado que propone Crispino, pero el factor determinante, el abuso, recae en la razón. Con esto no queremos decir que para Anselmo la razón no cumpla ninguna función con respecto al pecado en general y al del ángel en particular. Desde luego, ella también es decisiva pues, de no haber usado la razón, el Diablo -afirma el arzobispo- habría ignorado "qué debería o no debería querer" (DCD 22) y, en consecuencia, no habría sido injusto al no quererlo ni justo al quererlo, así como tampoco habría sido justa ni injusta su voluntad si no hubiera podido querer más que la justicia (véase $D C D$ 14). En otras palabras, la agencia moral requiere que el agente sea capaz de reconocer aquello que debe querer y, además, que pueda querer algo diferente de eso que sabe que debe -como hemos visto que sucede con la justicia y la felicidad-. Sin embargo, se debe admitir que, aun si insoslayable, el espacio que el $D C D$ le dedica a la razón queda eclipsado frente al de la voluntad pues, a los ojos de Anselmo, el pecado compete prin-

\footnotetext{
${ }^{17}$ En efecto, aunque Anselmo no utilice el término "abutor", en Monologion 71 Anselmo explica que el alma que desprecia a Dios es justamente castigada con la muerte "porque no se usó para lo que fue hecha" (quia se non utitur ad id ad quod facta est). Véase también Monologion 69 y 74; Proslogion 1; DV 2; DCD 7; y Agustín, De libero arbitrio III.1.3.
} 
cipalmente a esta última. ${ }^{18}$ Más aún, para él, el pecado consiste en un querer desordenado (inordinata voluntas).

Asumiendo una vez más el dato bíblico, esta vez, el pasaje de Isaías 14: 13-14, en el que el profeta le dice a Lucifer: "tú, que decías en tu corazón: '[...] seré semejante al Altísimo"', Anselmo explica que no es que el Diablo quería directamente ser igual a Dios, pues no pudo querer ser "lo que no pudo pensar" (DCD 4). El problema no radica en el objeto de deseo, en qué quiso, pues esto era la felicidad, un bien al que tendía por naturaleza. El querer ser igual a Dios es, en realidad, la consecuencia de cómo quiso, de la forma desordenada en que quiso. Es decir, cuando el Diablo abandonó la justicia en pos de aumentar su felicidad, lo que hizo fue querer por fuera de la voluntad de Dios; quiso algo de manera independiente (propria voluntate) sin sumisión alguna, lo cual le corresponde solo a Dios. Si, pues, se dice que quiso ser semejante a Dios fue porque quiso querer como Él. Pero además, al querer que prevaleciera su voluntad por sobre la de Dios, quiso ser más que Él, invirtiendo así la jerarquía ontológica; pecando, pues, de soberbia (véase DCD 4). ${ }^{19}$

Crispino también entiende que el Diablo pecó de soberbia y, por tanto, su explicación, al igual que la de Anselmo, redunda en un pretender ser causa de sí. No obstante, las mismas diferencias respecto de Anselmo que hemos venido señalando son las que hacen que deba interpretar el pasaje de Isaías de otra manera. ${ }^{20}$ Por una parte, al entender el pecado como un abuso voluntario de la razón, no solo debe integrar el aspecto racional en su explicación sino que este debe ocupar un lugar central; por otra, no puede decir que la caída se debió a preferir la felicidad a la justicia, pues entiende que stare in veritate es equivalente a stare in beatitudo. En consecuencia, según Crispino, el Diablo quiso permanecer en la felicidad en la que se hallaba -lo cual en modo alguno le estaba prohibido pero no porque tendiera a ella

\footnotetext{
${ }^{18}$ King sostiene que la innovación de Anselmo respecto de Agustín -su principal fuente- es mostrar a Lucifer como un agente moral plenamente calificado y es por ello que, a diferencia del Hiponense, incorpora el tema de la racionalidad del ángel. No obstante, advierte que aun cuando hay "interesting questions about moral knowledge [...] Anselm does not take them to be at issue in the case of primal sin”, (2012: 275, nota 34). Por su parte, Crispino también se refiere al requisito de conocimiento para la agencia moral señalando que el Diablo no podría haber sido castigado con justicia de haber sido creado ignorante. Véase DAP 4 y 14-17. ${ }^{19}$ Díez sintetiza con elocuencia los momentos del querer diabólico: "en su caída, el ángel [...] puso su voluntad 'debajo', 'en igualdad' y 'sobre' el querer divino”, Díez 2014: 106. Véase también Castañeda 2005: 50-54.

${ }^{20}$ Evans también nota la diferencia en este punto, pero no se la atribuye al mismo desarrollo teórico de Crispino, sino que sugiere que es la mera consecuencia de evitar las sutilezas de la explicación de Anselmo. Véase 1986: xxxv.
} 
naturalmente, como en el caso de Anselmo, sino porque permanecer en la felicidad era precisamente "lo que era necesario querer" (DAP 7)- pero "quiso permanecer de manera perversa" (DAP 18), "poniendo por debajo lo que debe ser puesto por encima, y poniendo por encima lo que debe ser puesto por debajo" (DAP 16).Y aunque ese "prave voluit stare" sin dudas recoge la idea del querer desordenado de Anselmo -de manera que, una vez más al igual que sucede en el $D C D$, el problema no fue el objeto sino el modo- para Crispino, la perversidad de esta voluntad no consiste en querer algo por sí mismo ni tampoco nace sponte, como había enseñado Anselmo (DCD passim), sino que proviene de un error intelectual: el Diablo estimó "que no era lo que era y que era lo que no era, esto es, que él no recibió de Dios lo que había recibido, y que podía hacerse igual a Dios" (DAP 21), no porque quisiera querer como Él sino porque, al estimar que no provenía de Dios el bien que poseía, se atribuyó "a sí mismo y no a Dios lo que tenía no por sí sino por Dios” (DAP 9), esto es, creyó que él, al igual que Dios, tenía la potestad de otorgar un bien. En pocas palabras, no fue una voluntad espontánea sino una estimación errónea la que lo condujo a querer "destruir su omnipotencia" y "hacer que Dios no fuera de quien todo proviene, por quien todo y en quien todo es" (Sermo in ramis palmarum 3). En suma, su pecado se debió a un uso efectivo de la razón, pero uno errado e indebido (un abusus) que lo condujo no solo a omitir su fin propio - amar a Dios-sino, incluso, a invertirlo.

Nótese que a pesar de las diferencias exegéticas, ambos autores coinciden en que, en última instancia, el pecado del Diablo consistió en negar su naturaleza ab alio. Pero hasta aquí llega la coincidencia. En el caso de Anselmo, esta negación ocurre no por el simple el hecho de tener una voluntad propia, pues también el mérito de los ángeles confirmados se debe a la aseidad de su voluntad; ocurre porque, a diferencia de ellos, que se dieron a sí mismos la justicia pero conservando aquella que en un primer momento habían recibido de Dios, la autodeterminación de la voluntad diabólica fue absoluta lo cual, como hemos dicho, es propio de Dios. ${ }^{21}$ Crispino, en cambio, entiende la cuestión de la aseidad casi literalmente: el Diablo cree que fue él quien se dio a sí mismo su propio bien, estimación falsa que, por otra parte, no es un elemento teórico menor en la economía del $D A P$. En primer lugar, porque le permite a Crispino retomar y explicar la segunda parte del pasaje de Juan que desencadena el diálogo. En efecto, si, como afirma Interrogatio apenas comenzado el $D A P$ siguiendo la definición anselmiana, estar en la falsedad es

${ }^{21}$ De allí que Goebel hable de una "aseité radicale" o "révolutionnaire" y de una "aseité conservatrice" (véase 2017: 88-90). 
"estimar que es lo que no es, o que no es lo que es", el Diablo no solo es un mentiroso sino que, como afirma el evangelista, es "el padre de la mentira" (Jn. 8: 44) por el hecho de haber sido el primero en concebirla (véase $D A P$ 22). En segundo lugar, porque es lo que le permite a Crispino integrar el elemento volitivo del pecado en un cuadro más amplio que incluye y destaca el costado racional de las creaturas capaces de pecar.

Así, la respuesta a la pregunta inicial de Interrogatio, por qué el Diablo no permaneció en la verdad de que Dios es mayor que él, se responde diciendo que no lo hizo porque no quiso, y no quiso porque abusó de la razón, creyendo erróneamente que podía hacerse igual a Dios. Posiblemente, la célebre explicación con la que Anselmo pone fin a la cuestión del pecado del ángel, "no quiso sino porque quiso" (DCD 27), le haya resultado insuficiente.

\section{Conclusión}

T a inquietud que motivó este trabajo había surgido de las dos

Lcaracterizaciones, en gran medida opuestas, que Evans había hecho del $D A P$, recordemos: que este sigue "punto por punto y tópico por tópico" el $D C D$, y que es una obra que se sostiene "por sus propios méritos". A la luz del análisis precedente debemos concluir que ambas afirmaciones son correctas, al menos en lo que toca a las primeras $42 \mathrm{sec}-$ ciones, pues a ellas hemos restringido la comparación.

La influencia del $D C D$ sobre el $D A P$ es innegable. El hecho de que sean los mismos pasajes bíblicos los que disparan los momentos centrales de sendos tratados podría no ser más que una semejanza superflua si no fuera que ellos determinan el contenido temático-problemático, por una parte, y el esquema argumentativo, por otra. No obstante, la concepción del dinamismo anímico que Gilberto expone en el $D A P$ y que es coherente con el resto de sus obras -lo cual nos permite inferir que este tratado puntual no responde a una simplificación deliberada para sortear las sutilezas teóricas de Anselmo (véase supra) - difiere en gran medida de la de su maestro.

$\mathrm{Al}$ poner el uso de la razón en el centro de su concepción ética y anímica, Gilberto se aleja no solo de Anselmo sino incluso de la tradición agustiniana propia del ámbito monástico. En efecto, mientras que esta siempre trató el problema del pecado poniendo el acento en la voluntad o, si se prefiere, en la orientación volitiva de las creaturas racionales, Gilberto sitúa el drama moral angélico y humano en el uso y el abuso de la razón de las creaturas con libre albedrío. Desde luego, no excluye el aspecto volitivo -así como ni Anselmo ni Agustín excluyen el aspecto racional/intelectual en sus respectivas teorías- pero tampoco lo analiza. Antes bien, tiende a darlo 
por supuesto, como en el caso de la definición de pecado a la que llega a través del estudio del libre albedrío y de la razón. De todas maneras, este alejamiento no debe entenderse como una ruptura, sino más bien como un cambio de énfasis en el que, por su parte, pueden apreciarse los nuevos aires intelectuales que pronto las escuelas urbanas contribuirían a extender por toda la Europa occidental.

\section{BIBLIOGRAFÍA}

Abulafia, A. S. y Evans, G. R. (1986), The Works of Gilbert Crispin, Abbot of Westminster (London: Oxford University Press).

Briancesco, E. (1978), “¿Cómo interpretar la moral de san Anselmo?”, Revista Latinoamericana de Filosofía, 4(2): 119-140.

Briancesco, E. (1982), Un triptyque sur la liberté. La doctrine morale de saint Anselme. De Veritate, de Libertate Arbitrii, De Casu Diaboli (Paris: Desclée de Brouwer).

Brouwer, C. y Gilon, O. (2017) (coords.), Liberté au Moyen Âge (Paris:Vrin).

Castañeda, F. (2005), "Estudio preliminar", en Anselmo de Canterbury: sobre la caída del Demonio (Bogotá: Universidad de los Andes, 2-133).

Corti, E. (2011), "Anselmo de Canterbury, De veritate: Veritas signata et veritas significans", Cuadernos del Sur, 40: 69-82.

Corti, E. (2013), “Malum y nihil: ¿son sinónimos en Anselmo de Canterbury?”, en El mal en la Filosofía Medieval (Buenos Aires: UNSAM edita, 79-100).

Crispino, G. (2017), Disputa de un cristiano con un gentil, traducción de N. Jakubecki (Buenos Aires: Centro de Investigaciones Filosóficas).

Davies, B. y Leftow, B. (2006) (eds.), The Cambridge Companion to Anselm (Cambridge: Cambridge University Press).

Disse, J. y Goebel, B. (2010) (eds.), Gott und die Frage nach dem Glück: Anthropologische und ethische Perspektiven (Frankfurt am Main: Knecht).

Díez, R. (2014), "Un aspecto de la unidad medieval en el De casu Diaboli de Anselmo", en S. Filippi y M. Coria (eds.), La identidad propia del pensamiento patrístico y medieval: ¿unidad y pluralidad? (Rosario: Paideia, 99-108).

Dreyer, M. (1997), “Veritas-rectitudo-iustitia: Grundbegriffe ethischer Reflexion bei Anselm von Canterbury", Recherches de theologie et philosophie Medievales, 64(1): 67-85.

Evans, G. R. (1980), Anselm and a New Generation (Oxford: Clarendon Press).

Evans, G. R. (1986), “Introduction - Section II.5”, en A. S. Abulafia y G. R. Evans, The Works of Gilbert Crispin, Abbot of Westminster (London: Oxford University Press).

Filippi, S. y Coria, M. (2014) (eds.), La identidad propia del pensamiento patrístico y medieval: ¿unidad y pluralidad? (Rosario: Paideia).

Goebel, B. (2010), “'Beatitudo cum iustitia': Anselm von Canterbury über Gerechtigkeit, Freiheit und das Verhältnis von Gerechtigkeit und Glück”, en J. Disse y 
B. Goebel (eds.), Gott und die Frage nach dem Glück: Anthropologische und ethische Perspektiven (Frankfurt am Main: Knecht, 60-120).

Goebel, B. (2012), "Vernunft und Autorität in den Religionsgesprächen Gilbert Crispins", Jahrbuch für Religionsphilosophie, 11:29-71.

Goebel, B. (2017), “'La justice sans laquelle elle n'est jamais libre': force, faiblesse et liberté de la volonté selon saint Anselme”, en C. Brouwer y O. Gilon (coords.), Liberté au Moyen Âge (Paris:Vrin, 79-100).

Hoffman, T. (2012) (ed.), A Companion to Angels in Medieval Philosophy (Leiden: Brill).

Jakubecki, N. (2018), "Divine Providence and Free Will in the De Angelo Perdito by Gilbert Crispin: sn Interpretation in Light of the Consolation of Philosophy", Bulletin de philosophie médiévale, 60: 9-28.

King, P. (2012) "Angelic Sin in Augustine and Anselm", en T. Hoffman (ed.), A Companion to Angels in Medieval Philosophy (Leiden: Brill, 261-282).

Novotný,V. (2014), Cur homo?: a history of the thesis concerning man as a replacement for fallen angels (Praga: Karolinum Press).

Olivares, I. (1952), Commentarii in universos Sancti Doctoris Anselmi, archiepiscopi Cantuariensis, et totius Angliae Primatis theologicos, dogmaticos, polemicos, scholasticos, Tractatus (1776-1790), según aparece consignado en Anselmo de Canterbury, De casu Diabili, editado por J. Alameda (Madrid: BAC).

Rakus, D. (1997), Towards an Anselmian Theodicy (Tesis doctoral para la Universidad de Toronto, Bibliothèque nationale du Canada).

Schmitt, F. S. (1946), S. Anselmi Cantuariensis Archiepiscopi Opera Omnia, vol. I (Edinburgh:Th. Nelson and Sons).

Schmitt, F. S. (1969) (ed.), Analecta Anselmiana: Untersuchungen über Person und Werk Anselms von Canterbury, vol. I (Frankfurt: Minerva).

Vanni Rovighi, S. (1969), "L'etica di S. Anselmo", en F. S. Schmitt (ed.), Analecta Anselmiana: Untersuchungen über Person und Werk Anselms von Canterbury, vol. I (Frankfurt: Minerva, 73-99).

Visser, S. y Williams, Th. (2006) “Anselm's Account of Freedom”, en B. Davies y B. Leftow (eds.), The Cambridge Companion to Anselm (Cambridge: Cambridge University Press, 179-203).

Whidden, D. (2015), “The Proslogion, Gilbert Crispin, and the Cur Deus homo:Anselm's Student and the Problems of the Incarnation", presentado en Reading Anselm, Context and Criticism (Boston College, 27-30 July). Inédito. URL $=<$ http:// www.academia.edu/14476066/The_Proslogion_Gilbert_Crispin_and_the_ Cur_Deus_homo_Anselm_s_Student_and_the_Problems_of_the_Incarnation>.

Zoppi, M. (2009), La verità sull'uomo: l'antropologia di Anselmo d'Aosta (Roma: Città Nuova).

Recibido: 03-06-2019; aceptado: 23-07-2019 